\title{
L'utopie délibérative de la mouvance antinucléaire et les paradoxes de son expérimentation
}

The deliberative utopia in the anti-nuclear movement and the paradoxes of its experimentation

Mikaël Chambru

\section{CpenEdition}

Journals

Édition électronique

URL : http://journals.openedition.org/communicationorganisation/5067

DOI : 10.4000/communicationorganisation.5067

ISSN : $1775-3546$

Éditeur

Presses universitaires de Bordeaux

Édition imprimée

Date de publication : 1 décembre 2015

Pagination : 61-72

ISSN : 1168-5549

Référence électronique

Mikaël Chambru, «L'utopie délibérative de la mouvance antinucléaire et les paradoxes de son expérimentation », Communication et organisation [En ligne], 48 | 2015, mis en ligne le 01 décembre 2018, consulté le 03 janvier 2020. URL : http://journals.openedition.org/communicationorganisation/ 5067 ; DOI : 10.4000/communicationorganisation.5067 


\title{
L'utopie délibérative de la mouvance antinucléaire et les paradoxes de son expérimentation
}

\author{
Mikoël Chombru'
}

Depuis les années soixante-dix, des dizaines de milliers de personnes se sont tour à tour engagées pour s'opposer au programme électronucléaire français imposé par le pouvoir politique au nom du rayonnement de la nation. Celui-ci a dû faire face, sans discontinuité depuis 1971, à des mobilisations contestataires, plus ou moins intenses, plus ou moins visibles et se déployant à des échelles variées au sein de l'espace public (Chambru, 2014). Au sein de cette activité protestataire " en train de se faire ", l'action collective est marquée par une adhésion des militants antinucléaires à une culture politique valorisant l'impératif délibératif, de telle sorte que ce dernier est une caractéristique structurante de leurs modes de fonctionnement sur le temps long. Cette exigence démocratique s'apparente une valeur utopique, dans la mesure où elle est à la fois une "fuite hors de la réalité » cherchant « à faire advenir un nouveau monde ", une « subversion sociale " ancrée dans le présent dénonçant la «crédibilité des systèmes d'autorité » et un « outil de mobilisation politique » cherchant à refonder l'ordre social institué (Dacheux, 2008 : 23-25). L'utopie est donc ici envisagée dans ses dimensions onirique, critique et politique.

Dès lors, considérant que l'utopie est l'un des moteurs de l'action collective et est au fondement du politique, les mouvements sociaux engagés dans la critique sociale de l'énergie électronucléaire proposent de "nouvelles réalités possibles» (Dacheux 2003 : 160). Au niveau des objectifs et des enjeux de la contestation d'abord, ils militent pour réintroduire des pratiques plus démocratiques dans le processus politique de développement de lénergie électronucléaire et tentent de faire advenir cette politisation et cette mise

1 ATER/Docteur en Sciences de l'information et de la communication, Université Grenoble Alpes, Groupe de recherche sur les enjeux de la communication (Gresec). Ses travaux de recherche interrogent les modalités de militantisme, d'expressions et d'actions publiques relatives à la publicisation des questions environnementales, scientifiques et techniques ; mikael.chambru@u-grenoble3.fr 
en discussion publique qu'ils appellent de leurs vœux (Chambru 2015). À l'intérieur même du mouvement, ensuite : les différentes composantes s'interrogent et expérimentent, en marge de l'espace public institué et de ces procédures codifiées, les meilleures modalités de discussions et de prises de décisions pour atteindre une forme idéale de fonctionnement approchant leurs idéaux délibératifs. Cette utopie délibérative de la mouvance antinucléaire est donc une utopie symbolique facteur de mobilisation, mais elle est aussi simultanément une utopie en actes : elle s'enracine dans les mondes vécus dans et par les pratiques concrètes des militants cherchant à la concrétiser au sein des espaces public oppositionnels. Ces derniers ne sont pas seulement les lieux symboliques où se déploient de façon abstraite les mouvements sociaux : ils sont aussi le lieu concret où se déploient les engagements individuels, et simultanément le lieu où se constituent les dimensions collectives de la militance antinucléaire. Son objectif d'arrêter le plus rapidement possible la production d'énergie électronucléaire et de construire une société où les dangers de cette dernière n'existeraient pas constitue d'ailleurs en lui-même une utopie animant ces mouvements sociaux.

En s'appuyant sur une vaste enquête de terrain réalisée dans le cadre de notre travail de recherche doctorale récemment achevé ${ }^{2}$, cet article interroge les modes d'échanges et de confrontations éprouvés au sein d'une frange de la mouvance antinucléaire que constitue le Réseau sortir du nucléaire. Lobjectif est d'analyser les tensions résultant de cette expérimentation singulière de l'utopie délibérative antinucléaire au sein d'une alliance inter-organisationnelle avec la dimension normative et plurielle de celle-ci, entre idéal inaccessible et idéal incarné, entre lieu imaginaire et modèle effectif. Il s'agit de dépasser le débat récurrent entre possibilité et impossibilité des utopies à se réaliser, afin de saisir celles-ci comme une composante vécue de l'action contestataire. En jeu, la compréhension du renouveau des pratiques démocratiques s'inventant au sein de ces formes d'organisation des mouvements sociaux (Suraud 2007 : 177).

\section{Le bricolage normatif d'un modèle délibératif}

Lors de la création du Réseau sortir du nucléaire en 1997, les militants antinucléaires bricolent un modèle délibératif singulier, à partir de leur utopie délibérative, des expériences passées d'instauration de celle-ci au sein des espaces publics oppositionnels et des normes instituées dans et par l'espace public institué. Cette opération de bricolage a pour objectif de créer des normes de communication et de délibération alternative en adéquation avec les valeurs éthiques défendues depuis plus de quatre décennies par la mouvance antinucléaire. Celles-ci s'appuient sur la prégnance d'un impératif délibératif

2 Celle-ci est composée de trente-huit entretiens semi-directifs réalisés avec des militants antinucléaires sur l'ensemble du territoire hexagonal, du dépouillement d'archives militantes récoltées par nos soins, d'une enquête ethnographique conduite pendant plusieurs mois au sein de plusieurs coalitions antinucléaires agissant à des échelles différentes (locale, régionale et nationale). 
recouvrant plusieurs dimensions - symbolique, identitaire, stratégique et pratique - s'entrecroisant les unes avec les autres. Symbolique tout d'abord : elle correspond à la perception et au sens que les militants antinucléaires donnent à la réalité sociale au sein de laquelle ils agissent, au regard de l'utopie qui anime leur engagement en faveur de cette cause. Identitaire ensuite : en s'appuyant sur la dimension symbolique des idéaux délibératifs, elle permet la construction sociale d'une identité collective partagée, elle fonctionne comme un marqueur dans le monde social. Puis, stratégique : elle correspond à un choix tactique d'organisation jugé comme le plus à même de permettre de répondre aux exigences de leur engagement que sont l'urgence, l'efficacité et la radicalité à agir ici et maintenant, face à leur adversaire. Et enfin pratique : elle est promue comme une condition nécessaire pour élaborer et appliquer des tactiques hétéroclites visant à publiciser le risque nucléaire au sein de l'espace public. Cette " culture politique " valorisant l'impératif délibératif et cherchant corollairement à la mettre en œuvre en interne est ancrée dans l'histoire d'une frange des mouvements sociaux (Neveu 2011 : 197).

Au sein du Réseau sortir du nucléaire, ce modèle délibératif visant à incarner et opérationnaliser les idéaux antinucléaires se matérialise par le choix d'articuler une certaine forme de centralisme jugée nécessaire par nombre de militants à la poursuite et à l'amplification de la lutte, et un refus de l'instauration d'une structure pyramidale. Sa mise en œuvre nécessite un important travail de négociation et de coalition conduit pendant plusieurs mois, via la rédaction d'une charte définissant le sens et les objectifs de cette nouvelle entité. Des règles du jeu sont ensuite instaurées afin de gérer cette alliance inter-organisationnelle. Pour les mouvements sociaux, l'utilisation de telles procédures pour organiser et structurer la délibération revêt un aspect stratégique de première importance : elle vise à limiter les épreuves de force et ainsi à maintenir la cohésion des coalitions contestataires. Ces règles du jeu sont institutionnalisées dans des statuts et dans un règlement intérieur que les militants s'engagent à respecter, encadrant ainsi de façon stricte les pratiques de discussion, de débat et de prise de décision. En ce sens, le Réseau sortir du nucléaire met en œuvre une conception procéduraliste de la délibération par l'usage d'un droit écrit, finalement assez proche des pratiques en vigueur au sein de certaines organisations anarchistes où ces enjeux démocratiques sont eux aussi centraux (Luck et Pereira 2011 : 106). Le but de cette démarche pragmatique est de réunir les militants antinucléaires autour d'une représentation commune et d'un projet collectivement partagés, afin d'inventer un modèle délibératif concret et opérationnel transmettant simultanément les valeurs utopiennes portées par les militants antinucléaires. Bien que diverses et variées, ces aspirations délibératives ont en commun, d'une part l'instauration d'un mode de fonctionnement horizontal et décentralisé plutôt que vertical et centralisé, d'autre part des modalités de discussion basées sur la participation et la délibération plutôt que sur la délégation et la 
représentation, et enfin un processus de décisions s'appuyant sur le consensus plutôt que sur le vote majoritaire.

Indispensable pour mettre en œuvre les idéaux délibératifs antinucléaires au regard des contraintes endogènes à la structuration de mouvance ellemême, la traduction pratique et juridique de ces derniers correspond à un dévoiement inévitable de ces idéaux. Ils ne sont désormais plus seulement un lieu imaginaire et un idéal inaccessible, ils deviennent une utopie en actes s'enracinant dans les mondes vécus à partir des contingences temporelles et situationnelles propres au Réseau sortir du nucléaire. Cette formalisation de l'utopie délibérative s'effectue aussi au détriment d'une souplesse organisationnelle bien souvent à l'œuvre au sein des coalitions locales, ainsi qu'au détriment de la participation du plus grand nombre à la délibération. La conduite quotidienne de l'organisation est en effet restreinte à un nombre réduit de militants regroupés au sein d'un Conseil d'administration, auquel l'ensemble des militants antinucléaires délèguent chaque année leur pouvoir de décision. Il est censé agir en respectant les prescriptions normatives du règlement intérieur et à partir des orientations définies par l'assemblée générale annuelle de laquelle il puise sa légitimité et de laquelle sont élus les administrateurs. Ce modèle délibératif s'appuie aussi sur une combinaison singulière de la critique de la délégation et de la représentation politique : l'abandon dans le processus décisionnel du consensus apparent et du principe d'unanimité pour le vote et le principe de la majorité d'une part, le recours à un processus de délégation de la délibération déconnecté du principe de représentation d'autre part. Ces choix visent à éviter les situations de blocage dans l'action du Réseau sortir du nucléaire, ainsi que les écueils des logiques représentatives présentes dans la majeure partie des organisations politiques et syndicales.

Sauf qu'en pratique, la légitimité de ce modèle délibératif bricolé se retrouve simultanément affaiblie par ces choix visant pourtant à opérationnaliser et concrétiser l'utopie délibérative des mouvements sociaux. Ils conduisent en effet à construire l'illégitimité des militants dépositaires du pouvoir de décision par délégation, du fait de leur non-représentativité de la diversité politique et idéologique de la mouvance antinucléaire. Ils conduisent également à l'émergence d'épreuves de force habituellement limitées au sein des espaces publics oppositionnels par l'utilisation de procédures de consensus, elles-mêmes héritées de l'attachement des militants antinucléaires au système de valeurs spécifiques qu'elles constituent dans leurs idéaux délibératifs. Ils limitent aussi singulièrement la participation des militants à la délibération, alors même que celle-ci continue d'être un référent normatif orientant les pratiques protestataires des militants. 


\section{L'emprise des procédures sur la participation}

Au sein du Réseau sortir du nucléaire, l'Assemblée générale annuelle est l'espace délibératif le plus ouvert à la participation aux décisions des acteurs de la protestation antinucléaire. Elle est par conséquent un moment concret de l'utopie délibérative des mouvements sociaux ouvrant, de part sa dimension expérimentale, le champ des possibles. Pour autant, la participation n'y est pas numériquement significative. En 2012 par exemple, elle n'a rassemblé qu'une centaine de personnes représentant soixante-dix coalitions sur les plus de neuf cent que revendique le Réseau sortir du nucléaire. Cette non-participation n'a rien d'étonnant, il s'agit d'un processus routinier au sein des mouvements sociaux où de tels espaces délibératifs sont institués pour gérer les alliances inter-organisationnelles (Mathieu 2011 : 58-62). Toutefois, les procédures de participation propres aux assemblées générales de cette coalition nationale, fortement encadrées et codifiées a priori, restreignent cette participation. Elles sont elles aussi autant marquées par les pratiques et les normes de l'espace public institué que par celles expérimentées par les mouvements sociaux au sein des espaces publics oppositionnels et des coalitions locales constituant sa base organisationnelle. En ce sens, cette utopie en actes ne se laisse pas réduire aux codifications délibératives de l'ordre social existant : elle est une brèche construisant de "nouvelles réalités possibles ", sans toute fois parvenir à s'en émanciper véritablement.

Premièrement, ne peuvent participer au maximum que deux militants des coalitions contestataires à jour de cotisation et ceux-ci ne sont pas censés s'exprimer en leur nom propre mais en tant que représentant de ces dernières. Ici encore, ce choix est à mettre en perspective avec la taille significative du Réseau sortir du nucléaire : le but est de faciliter le processus délibératif en réduisant le nombre de participants, quitte pour cela à ajouter un degré de délégation et à recourir à nouveau au principe de représentation. Aucune procédure ne régit par contre les modalités de désignation de ces délégués, chaque coalition étant libre de procéder comme elle le souhaite. La difficulté étant de trouver un militant disponible et prêt à s'engager dans cette tâche, ils sont généralement désignés sur la base du volontariat et ce sont bien souvent les mêmes personnes au fil des années. S'opère alors progressivement une distinction au sein même de la mouvance antinucléaire, entre d'un côté les militants habitués et experts $\mathrm{du}$ « jeu démocratique » de cet espace délibératif, et de l'autre ceux qui en sont exclus ou s'en excluent. La participation de nouveaux acteurs ne maîtrisant pas les procédures et les tactiques routinières de leurs contournements devient de plus en plus difficile. Ainsi, et à la différence de nombre de mouvements sociaux (Le Mazier 2013), les assemblées générales du Réseau sortir du nucléaire ne sont pas envisagées comme des lieux favorisant, par l'activité contestataire s'y déroulant, l'engagement de nouveaux individus en faveur de la cause antinucléaire. 
Deuxièmement, le déroulement concret de ces assemblées générales annuelles est lui aussi encadré par un certain nombre de procédures et de règles (minutage des discussions, nombre limité de prises de parole, gestion de l'attribution de la parole, etc) que les militants doivent respecter sans qu'il ne leur soit donné la possibilité de modifier en situation ce cadre normatif. Ayant pour but le déploiement d'une rationalité communicationnelle, cette procéduralisation donne à voir l'expérimentation d'une éthique de la discussion conceptualisée par la philosophie politique (Habermas 1999). Elle n'en reste pas moins profondément ambivalente en situation : elle permet une certaine égalité entre les militants, la participation d'un plus grand nombre d'entre eux aux discussions et la fluidité de celles-ci, tout en restreignant, de par son formalisme, les possibilités même de cette participation. Elle contribue également à déposséder les dynamiques délibératives d'une partie de leurs enjeux politiques. Les assemblées générales annuelles tendent en effet à se transformer en un lieu d'enregistrement et de validation de décisions déjà imaginées, discutées et quasiment validées par ailleurs : les dynamiques délibératives et la participation à celles-ci sont contraintes par les procédures. En ce sens, elles se démarquent des pratiques en vigueur au sein d'autres mouvements sociaux cherchant eux aussi à instaurer des conditions idéales d'échanges (Mouchard $2002: 136$ ).

Troisièmement, le cadre normatif du processus délibératif de ces assemblées générales annuelles est marqué par une forte asymétrie des ressources entre les différents participants et leurs statuts au sein du Réseau sortir du nucléaire. Une distinction nette est ainsi opérée entre les administrateurs et militants, mais aussi entre les militants. Ce sont les premiers qui décident a priori si les motions et campagnes proposées par les coalitions membres sont recevables ou non au regard des dispositions légales et réglementaires de l'organisation, quitte pour cela à s'appuyer sur la légitimité d'un professionnel du droit. Ce sont également eux qui établissent au préalable un ordre du jour non modifiable, qui siègent à la tribune, qui orchestrent le déroulement des échanges et qui veillent au déploiement des conditions idéales de parole. Cette emprise procédurale ne permet pas pour autant une stricte égalité et équité entre les militants, pas plus qu'elle n'efface les rapports de domination à l'œuvre dans ces interactions. Elle parvient au mieux à les encadrer et à les réduire, car elle ne restreint que partiellement l'occupation de la parole par les participants les plus aguerris à ce « jeu démocratique ». Du fait de la subsistance de niveaux de compétence différents entre eux et d'aspirations inégales à ne pas tenter d'imposer son point de vue, les militants ne sont donc pas égaux. En ce sens, les procédures délibératives apparaissent comme révélatrices d'une dissymétrie entre les militants et comme le résultat d'un processus collectif conflictuel.

En fin de compte, le déroulement de ces assemblées générales annuelles donne à voir un fort degré de procéduralisation et de routinisation du processus 
délibératif. Il révèle aussi la dimension agonistique de la matérialisation de l'utopie délibérative de la mouvance antinucléaire dans un modèle effectif. L'utopie en actes au sein du Réseau sortir du nucléaire est en effet tiraillé entre lieux imaginaires inaccessibles et tentatives d'expérimentation concrètes, ces dernières s'éloignant des précédents à mesure qu'elles s'enracinent dans des formes d'organisations collectives. Cette utopie antinucléaire n'est donc pas le modèle délibératif parfait qu'il ne resterait qu'à mettre en œuvre pour davantage de "démocratie ", elle n'est pas non plus le paradigme de société pour l'avenir, elle ne fait que fournir des pistes et des ouvertures vers les possibles.

\section{La mise en crise du modèle délibératif}

Malgré son caractère imparfait,ce modèle délibératif bricolé par les militants antinucléaires appelle des pratiques vécues - plus ou moins durables - qui fracturent et reconfigurent les «façons de faire collectif ». Il a aussi et surtout permis de maintenir la cohésion des différentes coalitions agrégées au sein du Réseau sortir du nucléaire jusqu'à ce que le licenciement d'un permanent de l'organisation, au printemps 2010, conduise à sa mise en crise. En dépit des idéaux partagés, sa dimension normative alors est critiquée de toutes parts. $\mathrm{Ne}$ pouvant plus appuyer sa légitimité sur un processus délibératif collectivement partagé, les décisions prises au sein de la coalition se retrouvent désormais systématiquement contestées par les militants étant en désaccord avec cellesci. En cause, le recours au principe du vote majoritaire pour trancher une décision collective clivante et la délégation de ce pouvoir de décision à un nombre circonscrit d'individus dont la légitimité ne repose pas sur le principe de représentation... c'est-à-dire les principes mêmes en vigueur depuis la création de cette coalition, ceux-là mêmes qui sont constitutifs du bricolage normatif opéré pour institutionnaliser l'utopie délibérative des militants antinucléaires. En ce sens, ce conflit fait émerger les tensions constitutives du modèle organisationnel du Réseau sortir du nucléaire. Elles sont d'autant plus violentes qu'elles ont été jusqu'alors occultées et qu'elles s'expriment ici en étant intriquées avec d'autres problématiques ayant peu à voir avec cette crise des normes délibératives, si ce n’est qu'elles en sont le révélateur.

Cette crise du modèle délibératif institué conduit assez rapidement à la remise en cause de la cohésion des différentes coalitions agrégées, affaiblissant ainsi le fonctionnement et les capacités d'action de l'organisation : le Réseau sortir du nucléaire se détache progressivement de l'identité stabilisée et publique d'un mouvement unifié partageant une communauté d'objectifs et de moyens. L'absence d'une posture délibérative consensuelle partagée exacerbe en effet les épreuves de force entre ses différentes composantes, mais aussi entre les différentes instances de l'organisation, entre l'assemblée générale annuelle, le conseil d'administration et l'équipe salariée. Face à l'impossibilité de dégager une position consensuelle, la crise se déplace dans l'arène judiciaire avec le 
dépôt, par les différents protagonistes du dossier, de plusieurs plaintes les uns contres les autres : la crise délibérative devient aussi une crise éthique, morale et politique. Elle fait en effet ressurgir des désaccords politiques clivants, ceux-là même qui avaient été temporairement dépassés lors de la création de l'organisation, entraînant la désagrégation de l'alliance inter-organisationnelle en une myriade de coalitions régionales et locales plus ou moins déconnectées les unes des autres.

De par son déroulement, les enjeux et les acteurs en jeu, cette crise délibérative mêlant des divergences de positionnement politique et de structuration organisationnelle se rapproche sensiblement de celle ayant eu lieu au sein d'autres mouvements sociaux tels qu'Agir ensemble contre le chômage (Mouchard 2002 : 143) ou Attac (Lusson 2007 : 119-121). Outre les aspects déjà évoqués, elle s'explique également par le succès grandissant d'une coalition nationale ne parvenant pas à s'adapter à la croissance de ses effectifs et aux enjeux délibératifs en découlant. Au fil des années, les instances nationales du Réseau sortir du nucléaire ont en effet pris une importance croissante dans le processus délibératif, sans que celle-ci ne résulte d'un choix explicitement formulé par l'ensemble des coalitions agrégées, au sein d'une assemblée générale par exemple. Ce processus d'institutionnalisation et de professionnalisation des mouvements sociaux est somme toute assez récurent au sein des dynamiques contestataires (Neveu 2011 : 95-96). Il se traduit par une centralisation de plus en plus importante de l'organisation, à contre-courant de l'utopie délibérative qu'elle porte depuis sa création. Cela devient ensuite d'autant plus conflictuel qu'est en jeu la formulation des choix stratégiques jusqu'alors unificateurs de la protestation : la nature des enjeux de la mobilisation, les choix d'identifications et les revendications portées dans l'espace public.

Pour tenter de trouver un nouveau compromis délibératif rassembleur, des mesures transitoires sont expérimentées, avec plus ou moins de difficultés, au fil des décisions prises lors des assemblées générales annuelles post-crises : modification du règlement intérieur et des statuts, création d'une commission de médiation et d'une commission de restructuration et de refondation, organisation de journées de réflexions stratégiques, procédures de consultation régulière des coalitions adhérentes, etc. Sans succès, car ces dispositifs participatifs n'ont en situation qu'un caractère consultatif dans le processus d'arrêt des décisions collectives au sein du Réseau sortir nucléaire, restreignant ainsi de fait leurs portées normatives en termes d'apport délibératif. Finalement, la décision est prise lors de l'assemblée générale annuelle du printemps 2015 de refonder radicalement, via l'organisation d'un congrès en 2016, le compromis délibératif sur lequel repose le fonctionnement de l'organisation. En jeu donc, le bricolage normatif d'un nouveau modèle délibératif visant à éviter les écueils de celui forgé en 1997... tout en traduisant dans ces nouvelles procédures les idéaux délibératifs des militants, ceux-là même ayant participé 
à la mise en crise de ce dernier. L'enjeu est de taille : dépasser tout d'abord la dimension émotionnelle de cette crise pour faire vivre l'utopie délibérative antinucléaire dans un « nouveau » modèle délibératif concret et opérationnel, (re)construire ensuite du sens et transmettre les valeurs éthiques et utopiques y étant rattachées, et enfin réunir la mouvance antinucléaire autour de ce projet et de cette représentation commune du Réseau sortir nucléaire. Il est aussi de première importance pour l'organisation en tant que forme sociale agencée au sein de l'espace des mouvements sociaux : parvenir à pérenniser durablement le Réseau sortir du nucléaire et lui redonner une place imminente au sein des dynamiques contestataires du programme électronucléaire, au regard du rôle moteur qu'il a pu avoir par le passé.

\section{La dimension utopienne des mouvements sociaux}

Le cas du Réseau sortir du nucléaire montre que l'utopie délibérative des mouvements sociaux contient deux dimensions insécables, que sont d'une part son aspect pratique, concret et opérationnel, et d'autre part sa temporalité et sa dynamique : elle est « synchroniquement les deux, désordonnée, irrégulière, le tout dans un équilibre mouvant et émulatif » (Cossette-Trudel 2010). Cette dimension utopienne des mouvements sociaux peut aussi être coûteuse et contraignante, notamment lorsque le modèle délibératif bricolé et institué est mis en crise par une part significative des militants y adhérant formellement jusqu'alors. Cette dissension entre idéal incarné et idéal inaccessible révèle tout d'abord les dimensions inévitablement antagonistes et conflictuelles des dynamiques délibératives des mouvements sociaux. Celles-ci sont en effet marquées par la tension entre les exigences des idéaux délibératifs des militants antinucléaires promouvant une éthique de la discussion et les pratiques effectives de ces derniers donnant à voir un respect plus ou moins marqué de ce principe en situation. Puis, elle interroge les possibilités concrètes de mise en œuvre de l'utopie délibérative des mouvements sociaux au regard des contraintes endogènes et exogènes aux mobilisations, notamment la hiérarchisation stratégique à effectuer entre les exigences normatives de délibération, d'efficacité et d'urgence d'agir face aux adversaires de la cause.

Ensuite, cette dissension entre lieu imaginaire et modèle effectif constitue simultanément l'un des moteurs de l'action collective antinucléaire, en témoigne l'attachement continu des militants à leurs idéaux délibératifs malgré le caractère imparfait de leurs expérimentations. Cette utopie délibérative régit en effet leurs pratiques communicationnelles et fait infatigablement figure d'impératif éthique à ne pas enfreindre, en ce sens qu'elle s'impose avec tout autant de consistance comme une norme orientant la critique sociale de l'énergie électronucléaire. Enfin, elle rappelle que les retentissements politiques des mouvements sociaux ne résident pas uniquement dans leurs capacités à peser sur le processus institutionnel mais aussi dans leurs dimensions empiriques et symboliques que sont la création de " communautés politiques élargies » et 
la promotion « un projet politique utopique : rendre le monde meilleur qu'il n'est " (Dacheux 2003 : 160-161). En ce sens, le Réseau sortir du nucléaire participe activement au processus de déroutinisation et de renouvellement permanent des structures et des thématiques de l'espace public contemporain.

\section{BIBLIOGRAPHIE}

CHAMBRU Mikaël (2014), «L'espace public à l'épreuve du phénomène antinucléaire en France (1962-2012) », ESSACHESS - Journal for communication studies, n 13, vol. 7 , p. 33-43.

CHAMBRU Mikaël (2015), « La critique du régime technopolitique des sciences par la mouvance antinucléaire : un éclairage sur le concept d'espace public oppositionnel ", Les enjeux de l'information et de la communication, $\mathrm{n}^{\circ}$ 16, vol. 3A, p. 29-38.

COSSETTE-TRUDEL Marie-Ange (2010), « La temporalité de l'utopie : entre création et réaction ", Temporalités, $\mathrm{n}^{\circ} 12$.

DACHEUX Eric (2003), «Associations et construction européenne : vers une société civile internationale ? ", Études de communication, n 26, p. 149-167.

DACHEUX Eric (2008), «Utopie et SIC. Pour une approche plurielle des démocraties contemporaines ", Communication, vol. 26, n 2, p. 9-44.

HABERMAS Jürgen (1999), De l'éthique de la discussion, Paris, Flammarion.

LE MAZIER Julie (2013), « Assemblée générale ». In BARBIER Rémi et al. (dir.), Dictionnaire critique et interdisciplinaire de la participation, Paris, GIS Démocratie et Participation.

LUCK Simon et PEREIRA Irène (2011), « Délibération et liberté politique dans les organisations anarchistes », Réfractions, $\mathrm{n}^{\circ} 27, \mathrm{p} .101-112$.

LUSSON Julien (2007), «Un nouvel élan pour Attac », Mouvements, n 49, p. 116123.

MATHIEU Lilian (2011), La démocratie protestataire, Paris, Les presses de SciencesPo.

MOUCHARD Daniel (2002), « Politique délibérative et logique de mobilisation. Le cas d'Agir ensemble contre le chômage ", Politix, n 57, p. 128-145.

NEVEU Catherine (2011), « Démocratie participative et mouvements sociaux : entre domestication et ensauvagement ? ", Participations, n 1, p. 186-209.

NEVEU Erik (2011), Sociologie des mouvements sociaux, Paris, La Découverte.

SURAUD Marie-Gabrielle (2007), « Communication ou délibération : les échanges dans la société civile ", Hermès, n 47, p. 177-184.

Résumé : Cet article propose d'analyser la place centrale qu'occupent les idéaux délibératifs des militants antinucléaires dans l'action des mouvements sociaux. Depuis son émergence, la mouvance antinucléaire expérimente, par le recours à des procédures plus ou moins contraignantes, ces exigences délibératives. En mettant la focale sur les modalités, les enjeux et les stratégies d'acteurs au sein du Réseau sortir du nucléaire, il s’agit plus précisément de décrypter les tensions résultant de ces expérimentations avec la 
dimension normative de cette utopie délibérative. En jeu, la compréhension du renouveau des pratiques démocratiques « en train » de s'inventer au sein des formes d'organisation des mouvements sociaux.

Mots-clés : espace public, mouvements sociaux, communication, délibération, participation, nucléaire.

Abstract : This article analyses the place occupied by the antinuclear militants' deliberative ideals in social movements since the seventies. Since its creations, the antinuclear movement uses of more or less restrictive procedures to experiments with such deliberative requirements. This article focuses on the methods, the challenges and the strategies of actors within Réseau sortir du nucleaire to highlight the tensions resulting from these experiments with the normative dimension of this deliberative utopia. It aims a providing a better understanding of the renewal of democratic practices currently invented within the different forms of organization of social movements.

Keywords : public sphere, social movements, communication, deliberation, participation, nuclear power. 
